\title{
Clinical Evaluation of Tumor Markers for Diagnosis in Patients with Non-small Cell Lung Cancer in China
}

\author{
Li Ma ${ }^{1}$, Xiao-Wei Xie ${ }^{2}$, Hai-Yan Wang ${ }^{2}$, Ling-Yun $\mathrm{Ma}^{2}$, Zhong-Guang Wen ${ }^{2 *}$
}

\begin{abstract}
Background: To evaluate the value of combined detection of serum carcinoembryonic antigen (CEA), cytokeratin 19 fragment (CYFRA21-1), and carbohydrateantigen 125 (CA125) for the clinical diagnosis of nonsmall cell lung cancer (NSCLC). Materials and Methods: Serum CEA, CYFRA21-1 and CA125 were assessed in 140 patients with NSCLC, 90 patients with benign lung disease and 90 normal control subjects, and differences of expression were compared in each group, and joint effects of these tumor markers in the diagnosis of NSCLC were analyzed. Results: Serum CEA, CYFRA21-1 and CA125 in patients with NSCLC were significantly higher than those with benign lung disease and normal controls $(P<0.05)$. The sensitivity of CEA, CYFRA21-1 and CA125 were $49.45 \%, 59.67 \%$, and $44.87 \%$ respectively. As expected, combinations of these tumor markers improved their sensitivity for NSCLC. The combined detection of CEA + CYFRA21-1 was the most cost-effective combination which had higher sensitivity and specificity in NSCLC. Elevation of serum CEA and CYFRA21-1 was significantly associated with pathological types $(P<0.05)$ and elevation of serum CEA, CYFRA21-1 and CA125 was significantly associated with TNM staging $(P<0.05)$. Conclusions: Single measurement of CEA, CYFRA21-1 and CA125 is of diagnostic value in the diagnosis of lung cancer, and a joint detection of these three tumor markers, could greatly improve the sensitivity of diagnosis on NSCLC. Combined detection of CEA + CYFRA21-1 proved to be the most economic and practical strategy in diagnosis of NSCLC, which can be used to screen the high-risk group.
\end{abstract}

Keywords: Non-small cell lung cancer - tumor markers - diagnosis

Asian Pac J Cancer Prev, 16 (12), 4891-4894

\section{Introduction}

Lung cancer has the highest mortality of any cancer worldwide. In China, lung cancer has the highest incidence, and it is the leading cause of mortality of all cancers. Nonsmall cell lung cancer (NSCLC) is increasing at a rapid rate, and both incidence and mortality are steadily growing (Hu et al., 2013). China will drive up global rates of this cancer in the foreseeable future (Chang et al., 2012; Liu et al., 2013; Lu et al., 2013).

Because NSCLC patients often do not exhibit specific symptoms, particularly in early stages, the majority of NSCLC patients are diagnosed already at advanced stages of the disease. Most of treatment outcomes of patients have been poor because the disease has already progressed to an advanced stage by the time it is diagnosed. Currently, conventional diagnostic tests such as chest radiographs, computed tomography (CT) scans, and fiber optic bronchoscopy (FB) are not sensitive enough for effective early detection. Meanwhile, the benign pulmonary nodules and malignant tumors cannot be distinguished by imaging methods currently (Henschke and Yankelevitz, 2008; Malik et al., 2013; Xie et al., 2013). Whereas, the pathological and cytological detections needed to obtain biopsy samples are invasive and difficult to repeat. Consequently, various tumor markers have been used to detect cancer at an early stage and monitor cancers.

Serum tumor markers are proteins that can be found in the blood, and their higher-than-normal concentrations have resulted in their widespread use in oncology (Ferrigno et al., 1994; Pamies and Crawford, 1996; Kav et al., 2012). Recent researches and clinical practices indicate that there are some tumor markers (TMs), including the carcinoembryonic antigen (CEA), cytokeratin 19 fragment (CYFRA21-1), and carbohydrateantigen 125 (CA125) are commonly found in NSCLC (Kav et al., 2012).

However, the sensitivity and specificity of one TM is low, but the combination of these TMs can improve the effectiveness of diagnosis of NSCLC. Because China is a developing country with a large population live in poor rural areas, the cost-effectiveness of each combination of these TMs is an important consideration. The present study was designed to evaluate the predictive value of serum levels of TMs for NSCLC and to find an optimal serum marker combination panel that benefits both patients and the medical insurance system. 


\section{Materials and Methods}

\section{Patients}

Institutional review board approval was obtained before conducting this study. Three groups of people were selected between January 1, 2012 and December 30, 2014 from the First Affiliated Hospital of PLA General Hospital. The first group comprised NSCLC patients. The diagnosis of each patient was confirmed by clinical outcome, imaging diagnosis and histological examinations. Stage and histological classification were evaluated according to the World Health Organization (WHO) 1999 lung cancer classification. All samples were collected before treatment. Cases with recurrence cancer were excluded. The second group was composed of patients with benign pulmonary diseases. In-patients with pneumonia, pleural effusion, bronchiectasia, and pulmonary abscess diagnoses were randomly selected. Patients were confirmed by routine standard diagnostic methods or histological examination, those patients with a history of malignant disease, digestive or kidney disease, or two or more concomitant lung diseases were excluded. The third group served as the healthy control group. Healthy people who took a healthy physical examination and all the examination in the normal range were included, except for those with a family history of lung cancer. Patients characteristics are presented in Table 1.

\section{Sample collection and detection}

Peripheral morning fasting blood were obtained from all study subjects. To isolate serum for subsequent testing, centrifugation was set as 2000 turn/RPM for 15 minutes. Chemiluminescence immunoassay was used to electrochemically detect CEA, CYFRA21-1 and CA125, and all experiment operating was in strict accordance with the instructions authorised by instrument and reagent kit. According to each manufacturer's recommendations, the positive cut-off values for each marker were $3.4 \mathrm{ng} / \mathrm{mL}$ for CEA, $3.3 \mathrm{ng} / \mathrm{mL}$ for CYFRA21-1, and $35 \mathrm{U} / \mathrm{mL}$ for CA125.

\section{Cost-effectiveness analysis}

The cost-effectiveness of the combination marker panel was evaluated in NSCLC group. The effectiveness is determined by the tumor marker sensitivity, and the cost depends on the expense that patients incur for the detection. According to the charge fee in the third class A level hospital in Bei jing, the cost for CEA, CYFRA21-1, and CA125 detection were each $¥ 100$.

\section{Statistical analysis}

Statistical analysis was performed using SPSS Statistics 19.0 (SPSS, Inc., Chicago, IL). The association between serum markers and NSCLC characteristics including stage and histological classification were compared by Student's t test. The data were described by means \pm standard deviation. The sensitivity, specificity of single markers and combination markers were calculated.

\section{Results}

Comparison of the CEA, CYFRA21-1 and CA125 Concentrations in the NSCLC Group, Benign Lung Disease Group, and Healthy Control Group

Serum level of CEA, CYFRA21-1 and CA125 in patients with NSCLC were significantly higher than those with lung benign disease or control $(P<0.05)$. This difference was not detected betweeen subjects with lung benign disease and healthy controls $(P>0.05)$ (Table 2$)$.

Comparison of the CEA, CYFRA21-1 and CA125 Concentrations among Each Clinicopathological Factor in the Cancer Group

Table 3 showed a significant increase in the concentration of CYFRA21-1 in squamous cell carcinoma, an increased CEA concentration in adenocarcinoma $(P$ $<0.05)$.

With respect to TNM stage, CEA, CYFRA21-1 and CA125 level was dramatically increased in stages III/IV when compared to stages I/II $(P<0.05)$ (Table 3$)$.

Comparison of the Sensitivity, Specificity of the Three Markers Individually and in Combination

The sensitivity of these three serum tumor markers was different when seperately conducted in the diagnosis for NSCLC, rank from high to low, CYFRA21-1 > CEA > CA125; regarding specificity, from high to

Table 1. Patients Characteristics

\begin{tabular}{lccc}
\hline & $\begin{array}{c}\text { NSCLC } \\
\text { group } \\
(140)\end{array}$ & $\begin{array}{c}\text { Pulmonary } \\
\text { benign disease } \\
\text { group (90) }\end{array}$ & $\begin{array}{c}\text { Normal } \\
\text { group } \\
(90)\end{array}$ \\
\hline Gender & & & \\
Male & 65 & 38 & 40 \\
Female & 75 & 57 & 50 \\
Age & $30 \sim 82$ & $23 \sim 80$ & $18 \sim 82$ \\
& $(53.5)$ & $(55.5)$ & $(54.5)$ \\
Histology & & & \\
Adenocarcinoma & 60 & & \\
Squamous cell lung cancer & 80 & & \\
NSCLC stages & & & \\
I/II & 55 & & \\
III/IV & 85 & & \\
Pulmonary benign disease & & 24 & \\
Pneumonia & & 22 & \\
Pleural effusion & & 15 & \\
Bronchiectasia & & 2 & \\
Pulmonary abscess & & 27 & \\
Phthisis & &
\end{tabular}

Table 2. Comparison of CEA, CYFRA21-1 and CA125 in Different Groups $(\chi \pm \mathrm{s}, \%)$

\begin{tabular}{|c|c|c|c|c|}
\hline Groups & Number & CEA (ng/ml) & CYFRA21-1 (ng/ml) & CA125 (U/ml) \\
\hline NSCLC group & 140 & $46.34 \pm 20.15$ & $24.56 \pm 12.68$ & $66.21 \pm 16.42$ \\
\hline Pulmonary benign disease & 90 & $5.15 \pm 2.86$ & $3.25 \pm 1.31$ & $22.37 \pm 8.67$ \\
\hline Healthy control & 90 & $4.38 \pm 2.47$ & $2.89 \pm 1.78$ & $18.95 \pm 6.48$ \\
\hline
\end{tabular}


Table 3. The Relationship between CEA, CYFRA21-1 and CA125 and the Clinicopathological Factors $(\chi \pm s, \%)$

\begin{tabular}{lcccc}
\hline Clinicopathological characteristics & $\mathrm{n}$ & CEA $(\mathrm{ng} / \mathrm{ml})$ & CYFRA21-1 $(\mathrm{ng} / \mathrm{ml})$ & CA125 $(\mathrm{U} / \mathrm{ml})$ \\
\hline Histological classification & & & & \\
$\quad$ Adenocarcinoma & 60 & $44.23 \pm 21.43$ & $4.21 \pm 1.12$ & $20.15 \pm 6.43$ \\
$\quad$ Squamous cell lung cancer & 80 & $6.15 \pm 2.54$ & $22.45 \pm 10.16$ & $19.33 \pm 7.17$ \\
NSCLC TNM stage & & & & $10.25 \pm 5.78$ \\
$\quad$ I/II & 55 & $24.25 \pm 12.46$ & $37.68 \pm 11.78$ & $40.34 \pm 16.63$ \\
III/IV & 85 & $65.27 \pm 16.64$ & & $78.43 \pm 20.53$ \\
\hline
\end{tabular}

Table 4. Sensitivity and Specificity (\%) of Tumor Marker and Its Combinations in Detecting Patients with NSCLC

\begin{tabular}{lcc}
\hline Tumor Marker & Sensitivity $(\%)$ & Specificity (\%) \\
\hline CEA & 49.45 & 71.23 \\
CYFRA21-1 & 59.67 & 90.52 \\
CA125 & 44.87 & 75.92 \\
CEA + CYFRA21-1 & 89.76 & 82.43 \\
CEA + CA125 & 76.24 & 79.34 \\
CYFRA21-1+ CA125 & 68.78 & 83.45 \\
CEA + CYFRA21-1+ CA125 & 90.54 & 74.21 \\
\hline
\end{tabular}

low, CYFRA21-1 > CA125 > CEA. In terms of joint detection, CEA + CYFRA21-1 + CA125 suggested to be a combination with highest sensitivity $(90.54 \%)$, followed by CEA + CYFRA21-1 (89.76\%). (Table 4).

\section{Cost-Effectiveness Analysis}

In the cost-effectiveness analysis, only the combination markers whose sensitivity exceeded $80 \%$ were included. The most cost-effective combination was CEA + CYFRA21-1 given that its cost was the lowest.

\section{Discussion}

Lung cancer has the highest mortality of any cancer worldwide and typically has a very poor prognosis. Lung cancer survival and therapy largely depend on the disease histology and stage at diagnosis. Early detection of lung cancer, and early treatment could significantly improve the response rate, and prolong survival time of patients (Parkin et al., 2005). Tumor markers, that are produced by tumor cells and released into blood or body fluids are considered to be associated with the process of tumorigenesis and tumor development (Hu et al., 2013; Lu et al., 2013). Detection of tumor markers is reported to be an useful tool in the diagnosis of lung cancer and could be an indicator for mornitoring curative effect as well as prognosis for patients with lung cancer (Ramshankar and Krishnamurthy, 2013). In this study, three common serum markers (CEA, CYFRA21-1 and CA125) in NSCLC were evaluated individually and in combination and our purpose is to develop a diagnostic system to improve sensitivity, and specificity on NSCLC screening.

CEA was first identified by GOLD and FREEDMAN in 1965 as an antigen specific for digestive tract adenocarcinomas (Hammarstrom, 1999). CEA is produced by the secretion cells of the normal adult gastrointestinal tract. It is a marker for monitoring colon and rectal cancers. Recently, CEA has become the marker of choice for lung adenocarcinomas (Foa et al., 1999). Meanwhile, several studies have reported increased CEA values in advanced bronchogenic cancers of various histological types (Rasmuson et al., 1983; Plavec et al., 2002). Generally, CEA levels vary in accordance with obvious changes in disease status, or they may precede their clinical recognition. Our data show that CEA has elevated levels and higher sensitivity in lung adenocarcinomas. Furthermore, it may have a role in monitoring therapy in advanced stages. In the present study, CEA is correlated with TNM stage and tumor invasion. In stages III/IV, CEA is observed at higher levels than in stages I/II.

CYFRA 21-1 is a sensitive tumor marker for NSCLC, particularly in squamous cell tumors. Because CYFRA 21-1 determines only fragments of cytokeratin 19, the test shows a higher specificity than tissue polypeptide antigen (TPA), which determines a mixture of cytokeratins 8,18 , and 19. CK-19 is a protein component of the intermediate filament protein in epithelial cells (Stieber et al., 1994). When epithelial cells transform into malignant cells, the keratin content is increased. Due to necrosis of tumor cells, the soluble fragment CYFRA21-1 of CK-19 is released into the blood. However, no organ tissue-specific and tumor-specific epithelial cytokeratins exist; therefore, it cannot be used as a tumor diagnosis indicator. However, CYFRA21-1 in serum will increase when epithelial cells transform into cancerous tumor cells, especially squamous epithelial cells of the lung and bladder transitional cells. Our study demonstrated that the sensitivity of CYFRA21-1 in detecting NSCLC was $59.67 \%$,the specificity was 90.52\%. Level of CYFRA21-1 in serum of patients with NSCLC were significantly higher than those with lung benign disease and healthy controls suggesting the CYFRA21-1 is important in early diagnosis of NSCLC.

Now, it is confirmed that CA125 is an important marker in diagnosis of lung cancer. In this study, serum CA199 in patients with NSCLC were significantly higher than those with lung benign disease and healthy controls $(P<0.05)$, suggesting CA125 is of importance in the diagnosis of patients with NSCLC.

Clinical and pathological research found that NSCLC is heterogeneous (Oguz et al., 2013). To improve the diagnostic sensitivity and specificity, severed tumor markers, combined together could be more effective in the diagnosis of NSCLC. Our results showed that CEA, CYFRA21-1 and CA125 as a single detection of NSCLC, CYFRA21-1 is a marker bearing the highest sensitivity with positive rate of $59.67 \%$. When CEA and CYFRA21-1 combined together, the sensitivity was $89.76 \%$, and specificity $82.43 \%$; most high sensitivity was $90.54 \%$ after a joint of CEA, CYFRA21-1 and CA125 together, 
and the specificity was $74.21 \%$.

The positive rates of CEA, CYFRA21-1 and CA125 were strongly related to TNM staging, consistent with other studies, and the levels of these TMs increased according to the progression of the tumor.

We detected a significant association between CEA and CYFRA21-1 and pathological types. The CYFRA21-1 was the most sensitive marker in squamous cell carcinomas, and CEA in adenocarcinoma.

Although the combination of tumor markers can improve the sensitivity, the specificity will decrease with increasing sensitivity, meanwhile, the cost will increase as well. China is a developing country, and therefore, the optimal cost should be considered. The best marker combination panel is useful not only for promoting the efficiency of diagnosis, but also for reducing the economic load for the patient and health management department. Some reports indicate that an analysis of cost-effectiveness is an appropriate evaluation of tumor marker combinations (Atherly and Camidge, 2012). In this study, we perform an analysis of cost-effectiveness for the tumor marker combinations. Hence, compared to other panels, the combination of CEA and CYFRA21-1 is the best choice to implement screening program in high-risk group.

In summary, single measurement of CEA, CYFRA21-1 and CA125 is of diagnostic value in the diagnosis of lung cancer, and a joint detection of these three tumor markers, could greatly improve the sensitivity of diagnosis on NSCLC. In addition, from an economic viewpoint, CEA and CYFRA21-1 might be a cost-effective combination for screening program.

\section{References}

Atherly AJ, Camidge DR (2012). The cost-effectiveness of screening lung cancer patients for targeted drug sensitivity markers. Br J Cancer, 106, 1100-6.

Chang S, Dai M, Ren JS, et al (2012). [Estimates and prediction on incidence, mortality and prevalence of lung cancer in China in 2008]. Zhonghua Liu Xing Bing Xue Za Zhi, 33, 391-4.

Ferrigno D, Buccheri G, Biggi A (1994). Serum tumour markers in lung cancer: history, biology and clinical applications. Eur Respir J, 7, 186-97.

Foa $P$, Fornier M, Miceli R, et al (1999). Tumour markers CEA, NSE, SCC, TPA and CYFRA 21.1 in resectable non-small cell lung cancer. Anticancer Res, 19, 3613-8.

Hammarstrom S (1999). The carcinoembryonic antigen (CEA) family: structures, suggested functions and expression in normal and malignant tissues. Semin Cancer Biol, 9, 67-81.

Henschke CI, Yankelevitz DF (2008). CT screening for lung cancer: update 2007. Oncologist, 13, 65-78.

$\mathrm{Hu}$ LA, Fu Y, Zhang DN, et al (2013). Serum IL-33 as a diagnostic and prognostic marker in non- small cell lung cancer. Asian Pac J Cancer Prev, 14, 2563-6.

Kav S, Tokdemir G, Tasdemir R, et al (2012). Patients with cancer and their relatives beliefs, information needs and information-seeking behavior about cancer and treatment. Asian Pac J Cancer Prev, 13, 6027-32.

Liu YC, Zhou SB, Gao F, et al (2013). Chemotherapy and late course three dimensional conformal radiotherapy for treatment of patients with stage III non- small cell lung cancer. Asian Pac J Cancer Prev, 14, 2663-5.

Lu YY, Huang XE, Xu L, et al (2013). Potential predictors of sensitivity to pemetrexed as first-line chemotherapy for patients with advanced non-squamous NSCLCs. Asian Pac J Cancer Prev, 14, 2005-8.

Malik PS, Sharma MC, Mohanti BK, et al (2013). Clinicopathological profile of lung cancer at AIIMS: a changing paradigm in India. Asian Pac J Cancer Prev, 14, 489-94.

Oguz A, Unal D, Tasdemir A, et al (2013). Lack of any association between blood groups and lung cancer, independent of histology. Asian Pac J Cancer Prev, 14, 453-6.

Pamies RJ, Crawford DR (1996). Tumor markers. An update. Med Clin North Am, 80, 185-99.

Parkin DM, Bray F, Ferlay J, et al (2005). Global cancer statistics, 2002. CA Cancer J Clin, 55, 74-108.

Plavec G, Ninkovic M, Kozlovacki G, et al (2002). [Tumor markers in pleural effusions in bronchogenic carcinoma and tuberculosis]. Vojnosanit Pregl, 59, 23-8.

Ramshankar V, Krishnamurthy A (2013). Lung cancer detection by screening - presenting circulating miRNAs as a promising next generation biomarker breakthrough. Asian Pac J Cancer Prev, 14, 2167-72.

Rasmuson T, Bjork GR, Damber L, et al (1983). Tumor markers in bronchogenic carcinoma. An evaluation of carcinoembryonic antigen, tissue polypeptide antigen, placental alkaline phosphatase and pseudouridine. Acta Radiol Oncol, 22, 209-14.

Stieber $P$, Dienemann H, Hasholzner U, et al (1994). Comparison of CYFRA 21-1, TPA and TPS in lung cancer, urinary bladder cancer and benign diseases. Int J Biol Markers, 9, 82-8.

Xie X, Zhao Y, Snijder RA, et al (2013). Sensitivity and accuracy of volumetry of pulmonary nodules on low-dose 16- and 64-row multi-detector CT: an anthropomorphic phantom study. Eur Radiol, 23, 139-47. 